\title{
Clinical Significance of CRP/ALB Ratio in Evaluating the Prognosis of Patients with DLBCL
}

\author{
Tiantian Shan*, Qiuyun Wang*, Zhen Cheng, Min Yan, Xiangtao Pan\# \\ Department of Hematology, Taicang Hospital Affiliated to Soochow University, Taicang, China \\ Email: "panxiangtao@csco.ac.cn
}

How to cite this paper: Shan, T.T., Wang, Q.Y., Cheng, Z., Yan, M. and Pan, X.T. (2021) Clinical Significance of CRP/ALB Ratio in Evaluating the Prognosis of $\mathrm{Pa}$ tients with DLBCL. International Journal of Clinical Medicine, 12, 403-414.

https://doi.org/10.4236/ijcm.2021.1210036

Received: September 3, 2021

Accepted: October 8, 2021

Published: October 11, 2021

Copyright $\odot 2021$ by author(s) and Scientific Research Publishing Inc. This work is licensed under the Creative Commons Attribution International License (CC BY 4.0).

http://creativecommons.org/licenses/by/4.0/

\begin{abstract}
Objective: Exploring the expression characteristics of CRP/ALB (CAR) in DLBCL patients and its value in prognostic judgment. Methods: We collected the basic information, clinical characteristics, laboratory examinations and follow-up prognosis of 142 newly diagnosed DLBCL patients with relatively complete data in our hospital and performed statistical analysis. We used Xtile analysis software to obtain the best cut-off value of CAR (0.33), compared the clinical characteristics and survival of patients in the high CAR group and the low CAR group, and compared the survival status with the IPI scoring system. Results: 1) There were significant differences in staging, grouping, IPI scores, extranodal involvement, LDH levels, $\beta 2$-microglobulin, CA125, and $\mathrm{Hb}$ levels between the high CAR group and the low CAR group (all $P<0.05$ ). 2) According to the survival curve, the OS of the high CAR group was significantly shorter than that of the low CAR group $(P<0.01)$, and the one-year, three-year and five-year survival conditions of high CAR group were all shorter than those of low CAR group. 3) COX analysis showed that high CAR is an independent poor prognostic factor for DLBCL patients. 4) A comparative analysis of OS, three-year and five-year survival showed that the combination of CAR and IPI was significantly better than the IPI system, and there was no significant difference in the evaluation value of the prognosis between CAR alone and IPI alone. Conclusion: High CAR value, like the IPI scoring system, is an independent poor prognostic factor of DLBCL, can be used as a reliable indicator of prognosis. And CAR can also be combined with IPI to evaluate the prognosis of DLBCL, of which the effect is better than that of IPI alone.
\end{abstract}


Keywords

DLBCL, CRP/ALB, IPI, Prognosis, Significance

\section{Introduction}

Diffuse large B cell lymphoma (DLBCL) is the most common lymphoma, a type of aggressive lymphoma [1]. According to current domestic and foreign guidelines [2], international prognostic index (IPI) scoring system is still the most commonly used prognostic scoring system for lymphoma such as DLBCL [2] [3] [4] [5]. Researches [6] [7] [8] have shown that many molecular genetic abnormalities, such as the abnormalities of genes BCL-6, BCL-2, P53, MYC and miRNA, are closely related to the poor prognosis of DLBCL. However, these scoring systems are not yet complete and must rely on complete imaging, serological examinations and general assessment of patients, and these examinations are timeconsuming, expensive, and limited by technology, so the clinical predictive value has certain limits. Therefore, clinicians need a simpler, faster, and more universal prognostic scoring system.

Recent studies have found [2] [3] [9] [10] [11] [12], tumor-related inflammation and the patient's nutritional status have a certain impact on the prognosis of many malignant tumors, including hematological tumors. Among them, the CRP/ ALB ratio composed of inflammation index C-reactive protein (CRP) and nutritional index albumin (ALB) is called CAR (C-reactive protein-to-Albumin Ratio, CAR), which can be used to assess the inflammation and nutritional status of tumor patients at the same time, closely related to the prognosis of tumors [13] [14] [15]. Therefore, this study used a comprehensive clinical analysis of 142 DLBCL patients to explore the characteristics of CAR expression in DLBCL and its relationship with prognosis, in order to evaluate the value of CAR for the prognosis of DLBCL.

\section{Materials and Methods}

\subsection{Case Selection}

We selected newly diagnosed DLBCL patients who were hospitalized in our hospital from March 1, 2009 to February 28, 2020, among them, a total of 142 cases had complete follow-up records and relatively complete data. Among the 142 cases, there were 80 males and 62 females, aged from 23 to 85 years (mean age 66.4 years). Each case was confirmed by immunopathological examination, and the diagnosis of DLBCL was confirmed according to the revised fourth edition of WHO classification criterion of lymphoid tissue tumors in 2016. Patients with a variety of acute or chronic infectious diseases, and those with rheumatoid arthritis, systemic lupus erythematosus or other rheumatic diseases and autoimmune diseases that could significantly affect the expression of CRP had been excluded. The patients were divided into two groups according to Hans, including GCB 
patients 67 cases (47.2\%) and non-GCB patients 75 cases $(52.8 \%)$.

This study has been discussed, approved and authorized by the Ethics Committee of Taicang Hospital affiliated to Suchow University. And the approval number was KY-2020-180.

\subsection{Research Methods}

\subsubsection{Grouping Method}

We collected cases' data of all patients, involving clinical indicators (such as gender, age, clinical stage, symptom grouping, IPI score, number of extranodal involvement, etc.) and laboratory indicators (such as LDH, $\beta 2-\mathrm{MG}, \mathrm{CA} 125, \mathrm{Hb}$, CRP and ALB, etc.). And then we used X-tile software to analyze to get the best cut-off value of the ratio of CRP and ALB (CAR value), which was used as the cutting point (the cut-off value of the two groups) to divide 142 subjects into high CAR group and low CAR group for comparative analysis.

\subsubsection{Follow-up Methods}

Follow-up was carried out by telephone inquiries, consulting medical records and other methods, and the dead cases were confirmed by consulting the original medical records, and the time of death or the last follow-up time was taken as the termination time of follow-up. The total end time of follow-up was December 31, 2020. The follow-up period was divided into three: 1 year, 3 years and 5 years. The overall survival (OS) was taken as the criterion. OS is defined as the course from the diagnosis of the disease to the death due to this disease or the last follow-up.

\subsection{Statistical Analysis}

We used X-tile software to get the best cut-off value of CAR, SPSS 23.0 software package for statistical analysis, expressed measurement data as mean \pm standard deviation, adopted t-test to compare between the two groups, expressed counting data as percentage (\%), adopted chi-square test for data comparison, adopted Pearson correlation analysis for correlation analysis, used COX risk model for multivariate analysis, and employed Kaplan-Meier method to draw survival curve. Furthermore, the receiver operator characteristic (ROC curve) and the areas under the curve (AUV) were compared by MedCalc software. The difference was statistically significant when $P<0.05$.

\section{Results}

\subsection{The Basic Condition of 142 Patients with DLBCL}

In 142 cases, 80 cases were males and 62 cases were females, aged from 23 to 85 years old, with an average age of 66.4 years old. According to clinical stages, 16 cases were in stage I, 47 cases were in stage II, 38 cases were in stage III, and 41 cases were in stage IV. Among them, there were 36 cases in group B (symptomatic group) and 107 cases in group A (asymptomatic group). According to the IPI score, there were 11 cases with 0 points, 37 cases with 1 point, 36 cases with 2 
points, 31 cases with 3 points, 17 cases with 4 points, and 10 cases with 5 points. According to the number of extranodal site involvement, there were 87 cases with 1 site involvement, 23 cases with 2,11 cases with 3,1 case with 4,1 case with 5, and 19 cases with no extranodal site involvement.

\subsection{Follow-Up Results}

All 142 cases were followed up to December 31, 2020. 139 cases (97.9\%) completed 1-year follow-up, 108 cases (76.1\%) completed 3-year follow-up, and 76 cases $(53.5 \%)$ completed 5-year follow-up. Until the follow-up deadline, among all the subjects, 99 cases (69.7\%) survived and 43 cases (30.3\%) died.

\subsection{The Relationship between CRP and ALB}

CRP is negatively correlated with ALB $\left(\chi^{2}=26.40, P<0.01\right)$. The specific results are shown in Table 1.

\subsection{Comparing in Groups by CAR (CRP/ALB Ratio)}

Using X-tile software to get the best cut-off value of CRP/ALB (CAR $=0.33)$, then we divided 142 patients into high CAR group (CAR $>0.33,64$ cases in total, accounting for $45.1 \%$ ) and low CAR group (CAR $<0.33$, 78 cases in total, accounting for $54.9 \%)$.

Patients' high CAR value is correlated with Ann Arbor stages III/IV $(P<$ $0.001)$, group B symptoms $(P<0.005)$, more than 1 site of extranodal focus $(P<$ $0.05)$, IPI scores $\geq 4(P<0.001)$, LDH elevation $(P<0.005), \beta 2-\mathrm{MG}$ elevation $(P$ $<0.001)$, CA125 elevation $(P<0.005)$ and anemia $(P<0.001)$. In addition, CRP levels increased $(100 \%)$ in all patients in the high CAR group, while ALB decreased by up to $42.2 \%$. The specific comparison results of clinical and hematological indexes between the two groups were shown in Table 2.

\subsection{Survival Analysis}

\subsubsection{Relationships between CAR, CRP, ALB and Prognosis of Patients with DLBCL}

Taking CAR $=0.33$ as the cut-off value to analyze its relationship with the prognosis of DLBCL, the result was statistically significant $(P=0.002)$, the median survival time of high CAR group was 15.05 months and that of low CAR group was 23.45 months. The median survival time of high CAR group was obviously shorter than the low CAR group. The specific results are shown in Figure 1. The specific results of 1-year, 3-year and 5-year survival analysis are shown

Table 1. The relationship between CRP and ALB.

\begin{tabular}{lrccc}
\hline \multirow{2}{*}{ Indexes } & & \multicolumn{2}{c}{ ALB } \\
\cline { 3 - 4 } & & normal & decrease \\
\hline \multirow{2}{*}{ CRP } & normal & 63 & 9 \\
& increase & 33 & 37 \\
\hline
\end{tabular}


Table 2. The comparison results of each index when CAR's (CRP/ALB) cut-off is 0.33 (statistical analysis by the number of cases).

\begin{tabular}{|c|c|c|c|c|}
\hline \multirow{2}{*}{ Indexes } & \multirow{2}{*}{ Specific indexes' data } & \multirow{2}{*}{$\begin{array}{l}\text { Low CAR group } \\
<0.33(\mathrm{n}=78)\end{array}$} & \multirow{2}{*}{$\begin{array}{c}\text { High CAR group } \\
>0.33(n=64)\end{array}$} & \multirow{2}{*}{$x^{2}$} \\
\hline & & & & \\
\hline \multirow{2}{*}{ Gender } & Male & 41 & 39 & \multirow{2}{*}{1.00} \\
\hline & Female & 37 & 25 & \\
\hline \multirow{2}{*}{ Age } & $\leq 60$ & 19 & 15 & \multirow{2}{*}{0.02} \\
\hline & $>60$ & 59 & 49 & \\
\hline \multirow[b]{2}{*}{ Stage } & $\mathrm{I} / \mathrm{II}$ & 45 & 18 & \multirow[b]{2}{*}{$12.45^{\star *}$} \\
\hline & III/IV & 33 & 46 & \\
\hline \multirow{2}{*}{ Group } & A & 66 & 40 & \multirow{2}{*}{$9.09^{* *}$} \\
\hline & B & 12 & 24 & \\
\hline \multirow{2}{*}{ IPI-A } & Low-medium low risk $(0$ - 2) & 58 & 26 & \multirow{2}{*}{$16.56^{* *}$} \\
\hline & Medium high-high risk $(\geq 3)$ & 20 & 38 & \\
\hline \multirow{2}{*}{ IPI-B } & Low-medium risk $(0-3)$ & 73 & 42 & \multirow{2}{*}{$17.85^{* *}$} \\
\hline & High risk $(\geq 4)$ & 5 & 22 & \\
\hline \multirow{2}{*}{ LDH } & Normal (109 - 245 U/L) & 53 & 26 & \multirow{2}{*}{$10.63^{* *}$} \\
\hline & Increase $(>245.0 \mathrm{U} / \mathrm{L})$ & 25 & 38 & \\
\hline \multirow{2}{*}{$\beta 2-\mathrm{MG}^{\Lambda}$} & Normal $(0.80-2.20 \mathrm{mg} / \mathrm{L})$ & 34 & 6 & \multirow{2}{*}{$19.48^{* *}$} \\
\hline & Increase $(>2.2 \mathrm{mg} / \mathrm{L})$ & 20 & 31 & \\
\hline \multirow{2}{*}{$\mathrm{CA} 125^{\wedge}$} & Normal $(0.0-35.0 \mathrm{U} / \mathrm{ml})$ & 58 & 29 & \multirow{2}{*}{$10.18^{* *}$} \\
\hline & Increase $(>35.0 \mathrm{U} / \mathrm{ml})$ & 10 & 20 & \\
\hline \multirow{2}{*}{$\begin{array}{l}\text { Extranodal } \\
\text { focus(es) }\end{array}$} & 1 & 64 & 42 & \multirow{2}{*}{$5.01^{*}$} \\
\hline & $\geq 2$ & 14 & 22 & \\
\hline \multirow{2}{*}{ Anemia } & Yes & 21 & 40 & \multirow{2}{*}{$24.42^{* *}$} \\
\hline & No & 57 & 24 & \\
\hline \multirow{2}{*}{ CRP } & Normal ( $\leq 10.0 \mathrm{mg} / \mathrm{l})$ & 72 & 0 & \multirow{2}{*}{$119.84^{* *}$} \\
\hline & Increase $(>10.0 \mathrm{mg} / \mathrm{l})$ & 6 & 64 & \\
\hline \multirow{2}{*}{ ALB } & Normal $(35.0-54.0 \mathrm{~g} / \mathrm{L})$ & 69 & 37 & \multirow{2}{*}{$17.45^{\star *}$} \\
\hline & Decrease $(<35.0 \mathrm{~g} / \mathrm{L})$ & 9 & 27 & \\
\hline
\end{tabular}

Note: 1$){ }^{\star} P<0.05,{ }^{* *} P<0.01$, the others are all $\left.P>0.05 .2\right) \boldsymbol{\Lambda}$ : Some cases' data of $\beta 2$-MG, CA125 at the initial diagnosis were missing, but all the follow-up treatment data were available. Considering the unity of the data, the actual data at the initial diagnosis prevailed in the analysis.

Table 3. The relationship between CAR and prognosis of DLBCL.

\begin{tabular}{|c|c|c|c|c|c|c|c|}
\hline & & \multicolumn{6}{|c|}{ Overall survival } \\
\hline \multicolumn{2}{|c|}{ Indexes } & \multicolumn{2}{|c|}{1 year } & \multicolumn{2}{|c|}{3 years } & \multicolumn{2}{|c|}{5 years } \\
\hline & & $<1$ & $\geq 1$ & $<3$ & $\geq 3$ & $<5$ & $\geq 5$ \\
\hline \multirow{3}{*}{ CAR } & $<0.33$ & 20 & 58 & 49 & 29 & 61 & 17 \\
\hline & $>0.33$ & 27 & 37 & 55 & 9 & 58 & 6 \\
\hline & $x^{2}$ & \multicolumn{2}{|c|}{$4.35^{\star}$} & \multicolumn{2}{|c|}{$9.59^{* *}$} & \multicolumn{2}{|c|}{$4.06^{*}$} \\
\hline
\end{tabular}

Note: ${ }^{*} P<0.05,{ }^{* *} P<0.01$. 


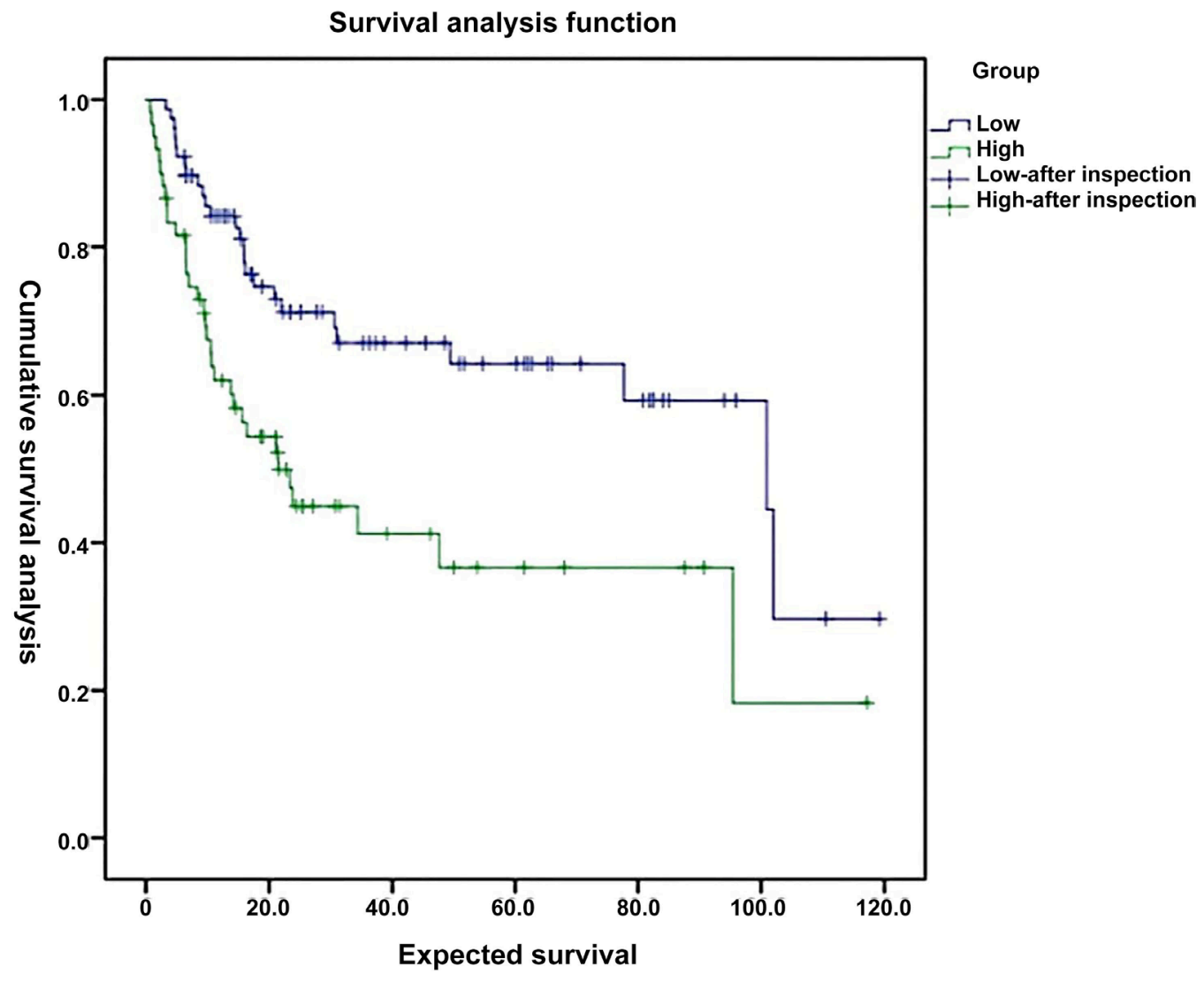

Figure 1. The relationship between CAR and survival and prognosis of DLBCL $(P=0.002)$.

in Table 3. It is shown from Table 3 that high CAR was associated with shorter overall survival of 1-year, 3-year and 5-year in patients with DLBCL.

\subsubsection{Analysis of the Relationships between Each Clinical Indexes and Prognosis of Patients with DLBCL}

\section{1) Univariate analysis}

Univariate analysis was conducted to analyze the relationships between overall survival and 15 indexes such as gender, age, stage and therapies containing Mabthera (R), etc. The results showed that overall survival was related to 6 indexes including the number of extranodal involvement, IPI, LDH, CRP, ALB and CAR. The specific results are shown in Table 4.

\section{2) Multifactor analysis}

Multivariate analysis was performed on 4 indicators of extranodal involvement, IPI, LDH, and CAR which were related to overall survival in univariate analysis. The results showed that IPI score (HR: 2.324, 95\% CI: $1.304-4.142, P=$ 0.000 ) and CAR (HR: 1.806, 95\% CI: 1.049 - 3.109, $P=0.030$ ) were both independent prognostic factors of DLBCL (considering that in univariate analysis, CRP and ALB were statistically significant, and the two indexes had been combined into one index of CAR, so there was no separate statistical analysis of these two indexes). Extranodal involvement $(P=0.62)$ and $\mathrm{LDH}(P=0.06)$ were no statistically significance. 
Table 4. Univariate analysis of the relationships between each clinical indexes and prognosis of patients with DLBCL.

\begin{tabular}{ccccc}
\hline Influencing factors & & HR & $95 \%$ CI & $P$ \\
\hline Gender & male & 0.892 & $0.531-1.499$ & 0.67 \\
Age & $>60$ & 1.441 & $0.763-2.718$ & 0.26 \\
Stage & III/IV & 1.490 & $0.878-2.529$ & 0.14 \\
Group & B & 1.501 & $0.850-2.651$ & 0.16 \\
Extranodal involvement & $>1$ & 1.881 & $1.081-3.275$ & $0.02^{*}$ \\
IPI score & high & 2.627 & $1.541-4.476$ & $0.00^{* *}$ \\
R therapy & no & 1.492 & $0.888-2.508$ & 0.13 \\
Anemia & yes & 1.572 & $0.939-2.629$ & 0.08 \\
LDH & increase & 1.915 & $1.146-3.200$ & $0.01^{* *}$ \\
$\beta 2-\mathrm{MG}$ & increase & 1.678 & $0.883-3.186$ & 0.11 \\
CA125 & increase & 1.389 & $0.759-2.540$ & 0.29 \\
CRP & increase & 2.066 & $1.224-3.485$ & $0.01^{* *}$ \\
ALB & decrease & 2.907 & $1.730-4.886$ & $0.00^{* *}$ \\
D-D & increase & 1.858 & $0.841-4.109$ & 0.13 \\
CAR & increase & 2.210 & $1.317-3.708$ & $0.00^{* *}$ \\
\hline
\end{tabular}

Note: ${ }^{\star} P<0.05,{ }^{*} P<0.01$, the other are all $P>0.05$.

\subsubsection{Comparison of Prognostic Significance of CAR Combined with IPI, IPI Alone and CAR on DLBCL}

We analyzed the prognosis of CAR combined with IPI and IPI alone, scored 1 point for high CAR and directly superimposed onto IPI score. And we evaluated the prediction accuracy by comparing the area under the receiver operating characteristic (ROC) curve of CAR combined with IPI, IPI alone and CAR. The results showed that no matter the overall survival condition (Figure 2(a)) or the three-year and five-year survival condition (Figure 2(c) and Figure 2(d)), the significance of IPI combined with CAR to prognosis was higher than IPI alone, that is, CAR combined with IPI had better prognostic value than IPI alone, while it had little effect on 1-year survival time (Figure 2(b)). Comparing the ROC curve of IPI with CAR, the area under the curve of CAR was larger than all that of IPI in 1 year, 3 years and 5 years, but the difference was not statistically significant.

\section{Discussion}

At present, the discussion on the relationship between inflammation and tumor has become a hot research topic [10] [16] [17]. On the one hand, tumor-related inflammation can mediate and inhibit tumor progression [18], on the other hand, it can also play a counterproductive role, stimulating tumor cell growth, proliferation and tumor angiogenesis, accelerating tumor invasion and metastasis [19] [20] [21]. As an accurate and reliable indicator of inflammation, CRP is 


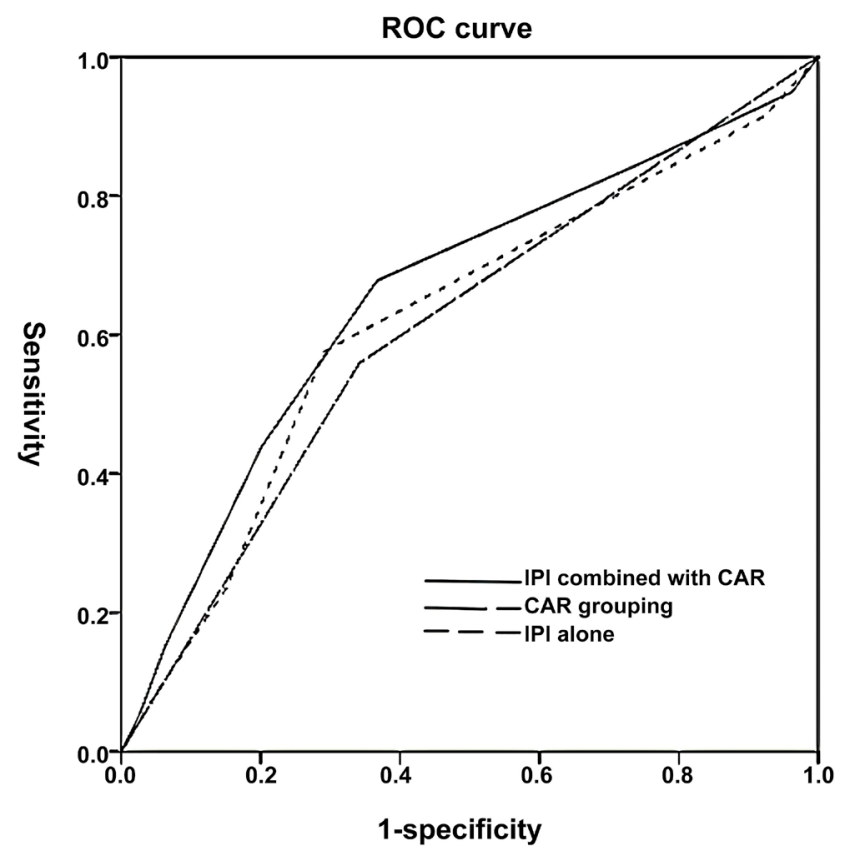

(a)

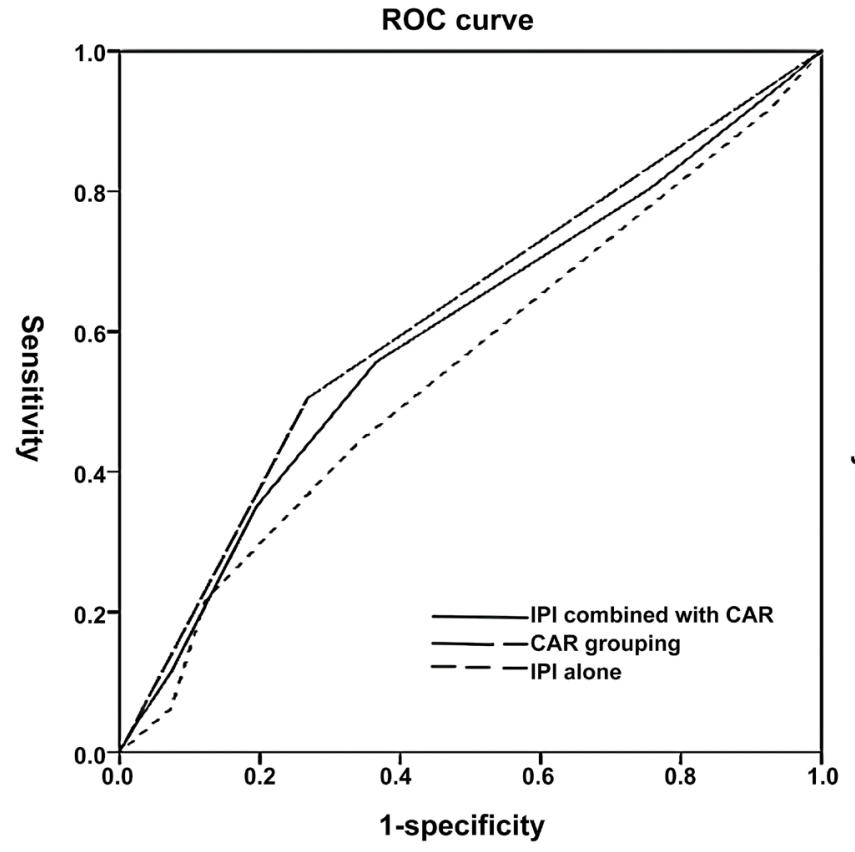

(c)

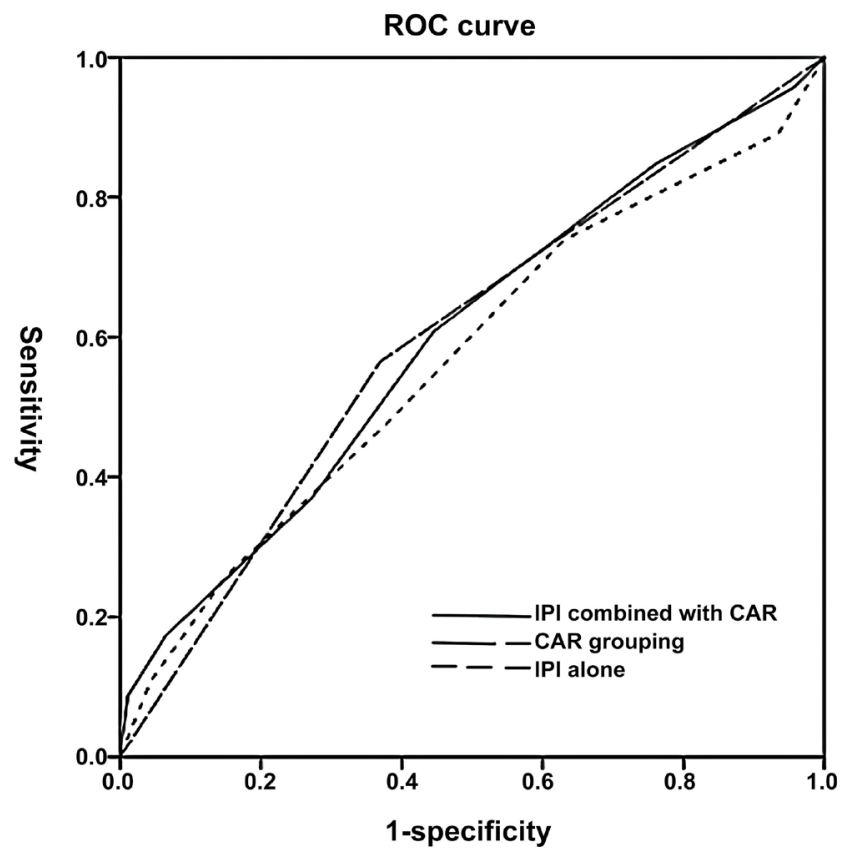

(b)

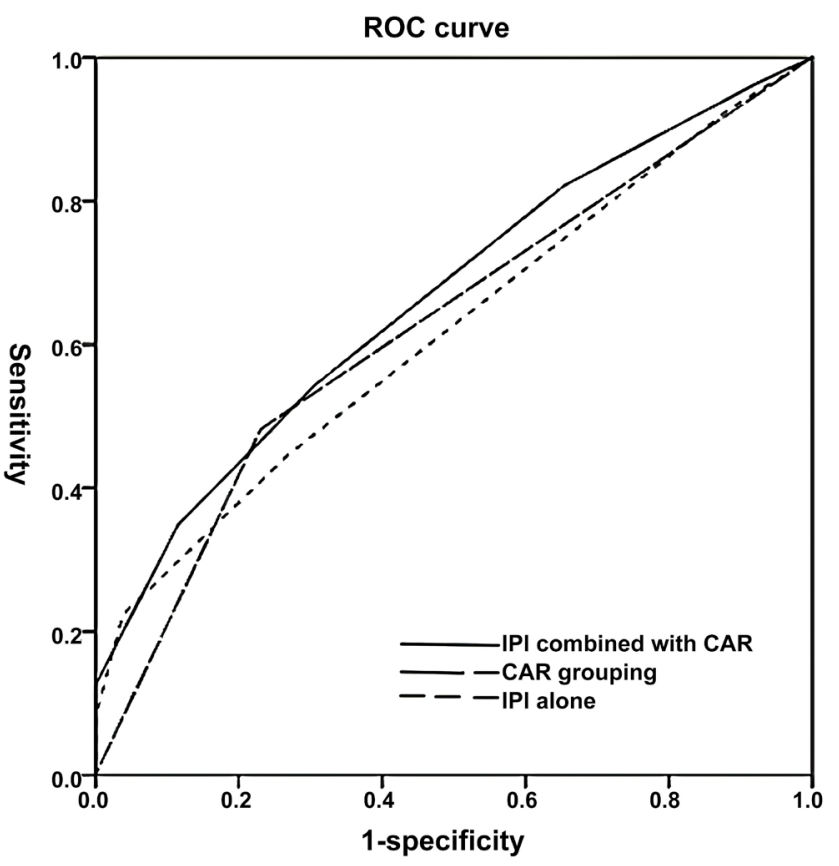

(d)

Figure 2. (a) ROC curve comparison of the prognostic value of CAR alone in overall survival condition (IPI combined with CAR versus IPI alone, $P=0.02$; IPI versus CAR, $P=0.86$ ); (b) ROC curve comparison of the prognostic value of CAR combined with IPI and IPI alone in 1-year survival condition (IPI combined with CAR versus IPI alone, $P=0.11$; IPI versus CAR, $P=0.68$ ); (c) ROC curve comparison of the prognostic value of CAR combined with IPI and IPI alone in 3-year survival condition (IPI combined with CAR versus IPI alone, $P=0.01$ and IPI versus CAR, $P=0.32$ ); (d) ROC curve comparison of the prognostic value of CAR combined with IPI and IPI alone in 5 -year survival condition (IPI combined with CAR versus IPI alone, $P=0.02$; IPI versus CAR, $P=0.91$ ).

produced by hepatocytes and epithelial cells under the stimulation of IL-6, IL-1, TNF and other inflammatory factors, which is not only related to the poor prog- 
nosis of tumors, but also increases the occurrence risk of future tumors [22] [23]. Researches [24] [25] [26] [27] have found that the association between increased circulating levels of CRP and increased cancer risk is not directly causal. In order to better predict the prognosis of tumors, the researchers proposed to combine CRP with ALB, which is an indicator of nutritional status, into a new indicator, CRP/ALB (CAR), to predict the prognosis of cancer patients [12] [13] [14] [28]. Domestic and foreign researches [12] [13] [14] [28] [29] [30] have proved that, in solid tumors such as pancreatic cancer, gastrointestinal tumors, lung cancer and reproductive system, CAR can independently predict the prognosis of patients.

This study took CAR $=0.33$ as the best critical value, which is basically consistent with the current domestic and foreign researches' CAR value of 0.03 0.68 [14] [15] [31]. We selected the data of patients at the time of initial diagnosis, which were more representative, stable and uniform, and can better judge the predictive value of CAR value at the time of initial diagnosis. In this study, the differences in staging, grouping, number of extranodal involvement sites, IPI score, LDH level, $\beta 2$ microglobulin, CA125, and anemia between the high CAR group and the low CAR group were statistically significant, which indicated that patients in the high CAR group had more serological abnormalities and more serious clinical symptoms risk [30]. Moreover, the OS of the high CAR group was significantly shorter than that of the low CAR group, and the median survival time was 8.4 months less in the lower CAR group. This suggested that high CAR level was closely related to the poor prognosis of DLBCL, of which the result was consistent with the results of solid tumors [29] [30] [32].

The single-factor and multi-factor COX survival analysis of CAR in DLBCL showed that both CAR and IPI scores were independent prognostic factors for DLBCL (CAR: $\mathrm{HR}=1.806, P=0.03$; IPI score: $\mathrm{HR}=2.234, P=0.00$ ). To further evaluate the prognostic value of CAR, this study also combined CAR and IPI scoring system to form a new scoring system to evaluate the prognosis of DLBCL. ROC curve results showed that CAR and IPI have no significant difference in the assessment of survival (the overall survival time, 1-year, 3-year and 5-year survival, $P$ all $>0.05)$. When assessing the overall survival and three-year and fiveyear survival of patients, CAR combined with IPI had a better predictive value for the prognosis of DLBCL patients than the IPI scoring system alone ( $P=0.02$, 0.01 and 0.02 , respectively).

Therefore, the CAR value, like the IPI scoring system, is an independent poor prognostic factor of DLBCL, which can be used as a reliable indicator to judge the prognosis, and it is easy to obtain. CAR combined with IPI scoring system has better prognostic value than IPI scoring system alone. However, this study is a single-center preliminary study, the number of DLBCL cases included is not too large, and the time span for selecting cases is also long, there may exist information bias, loss to follow-up, etc. Therefore, it needs to further expand the number of cases, extend follow-up time, conduct multi-center, prospective and other unified standards to further study. 


\section{Acknowledgements}

All authors read and approved the final manuscript.

\section{Conflicts of Interest}

No potential conflict of interest was reported by the author(s).

\section{References}

[1] Swerdlow, S.H., Campo, E., Pileri, S.A., et al. (2016) The 2016 Revision of the World Health Organization Classification of Lymphoid Neoplasms. Blood, 127, 2375-2390. https://doi.org/10.1182/blood-2016-01-643569

[2] Zhou, Z., Sehn, L.H., Rademaker, A.W., et al. (2014) An Enhanced International Prognostic Index (NCCN-IPI) for Patients with Diffuse Large B-Cell Lymphoma Treated in the Rituximab Era. Blood, 123, 837-842.

https://doi.org/10.1182/blood-2013-09-524108

[3] Joffe, L., Dwyer, S., Glade Bender, J.L., et al. (2019) Nutritional Status and Clinical Outcomes in Pediatric Patients with Solid Tumors: A Systematic Review of the Literature. Seminars in Oncology, 46, 48-56. https://doi.org/10.1053/j.seminoncol.2018.11.005

[4] Ngo, L., Hee, S.W., Lim, L.C., et al. (2008) Prognostic Factors in Patients with Diffuse Large B Cell Lymphoma: Before and after the Introduction of Rituximab. Leukemia \& Lymphoma, 49, 462-469. https://doi.org/10.1080/10428190701809156

[5] (1993) A Predictive Model for Aggressive Non-Hodgkin's Lymphoma. The New England Journal of Medicine, 329, 987-994. https://doi.org/10.1056/NEJM199309303291402

[6] Li, L., Zhang, X., Zhang, T., et al. (2018) Prognostic Significance of BCL-2 and BCL-6 Expression in MYC-Positive DLBCL. Clinical Lymphoma, Myeloma \& Leukemia, 18, e381-e389. https://doi.org/10.1016/j.clml.2018.06.010

[7] Huang, P., Chen, S., Yang, X., et al. (2019) Prognostic Evaluation of P53 and BCL2 Proteins in MYC/BCL2 Double Expression DLBCL. Chinese Journal of Hematology, 40, 589-593.

[8] Sun, R., Zheng, Z., Wang, L., et al. (2021) A Novel Prognostic Model Based on Four Circulating miRNA in Diffuse Large B-Cell Lymphoma: Implications for the Roles of MDSC and Th17 Cells in Lymphoma Progression. Molecular Oncology, 15, 246261. https://doi.org/10.1002/1878-0261.12834

[9] Taniguchi, K. and Karin, M. (2018) NF- $\kappa$ B, Inflammation, Immunity and Cancer: Coming of Age. Nature Reviews Immunology, 18, 309-324.

https://doi.org/10.1038/nri.2017.142

[10] Coussens, L.M. and Werb, Z. (2002) Inflammation and Cancer. Nature, 420, 860-867. https://doi.org/10.1038/nature01322

[11] Jiang, Z., Li, Y., Han, G., et al. (2016) Association of Serum Albumin Level with Clinicopathologic Features and Prognosis in Colon Cancer. Chinese Journal of Gastrointestinal Surgery, 19, 80-83.

[12] Bairey, O., Shacham-Abulafia, A., Shpilberg, O., et al. (2016) Serum Albumin Level at Diagnosis of Diffuse Large B-Cell Lymphoma: An Important Simple Prognostic Factor. Hematological Oncology, 34, 184-192. https://doi.org/10.1002/hon.2233

[13] Kinoshita, A., Onoda, H., Imai, N., et al. (2015) The C-Reactive Protein/Albumin Ratio, a Novel Inflammation-Based Prognostic Score, Predicts Outcomes in Patients 
with Hepatocellular Carcinoma. Annals of Surgical Oncology, 22, 803-810. https://doi.org/10.1245/s10434-014-4048-0

[14] Wu, M., Guo, J., Guo, L., et al. (2016) The C-Reactive Protein/Albumin Ratio Predicts Overall Survival of Patients with Advanced Pancreatic Cancer. Tumor Biology, 37, 12525-12533. https://doi.org/10.1007/s13277-016-5122-y

[15] Zhou, T., Zhan, J., Hong, S., et al. (2015) Ratio of C-Reactive Protein/Albumin Is an Inflammatory Prognostic Score for Predicting Overall Survival of Patients with Small-Cell Lung Cancer. Scientific Reports, 5, Article No. 10481. https://doi.org/10.1038/srep10481

[16] Cheng, Z., Yan, M., Lu, Y., et al. (2020) Expression of Serum BMP6 and Hepcidin in Cancer-Related Anemia. Hematology, 25, 134-138. https://doi.org/10.1080/16078454.2020.1738098

[17] Karin, M. (2006) Nuclear Factor-kappaB in Cancer Development and Progression. Nature, 441, 431-436. https://doi.org/10.1038/nature04870

[18] Mei, Z., Liu, Y., Liu, C., et al. (2014) Tumour-Infiltrating Inflammation and Prognosis in Colorectal Cancer: Systematic Review and Meta-Analysis. British Journal of Cancer, 110, 1595-1605. https://doi.org/10.1038/bjc.2014.46

[19] Wang, X. and Lin, Y. (2008) Tumor Necrosis Factor and Cancer, Buddies or Foes? Acta Pharmaceutica Sinica, 29, 1275-1288. https://doi.org/10.1111/j.1745-7254.2008.00889.x

[20] Singh, N., Baby, D., Rajguru, J.P., et al. (2019) Inflammation and Cancer. Annals of African Medicine, 18, 121-126. https://doi.org/10.4103/aam.aam 5618

[21] Liao, C.P., Booker, R.C., Brosseau, J.P., et al. (2018) Contributions of Inflammation and Tumor Microenvironment to Neurofibroma Tumorigenesis. Journal of Clinical Investigation, 128, 2848-2861. https://doi.org/10.1172/JCI99424

[22] Heikkilä, K., Ebrahim, S. and Lawlor, D.A. (2007) A Systematic Review of the Association between Circulating Concentrations of $\mathrm{C}$ Reactive Protein and Cancer. Journal of Epidemiology and Community Health, 61, 824-833. https://doi.org/10.1136/jech.2006.051292

[23] Allin, K.H., Bojesen, S.E. and Nordestgaard, B.G. (2009) Baseline C-Reactive Protein Is Associated with Incident Cancer and Survival in Patients with Cancer. Journal of Clinical Oncology, 27, 2217-2224. https://doi.org/10.1200/JCO.2008.19.8440

[24] Chaturvedi, A.K., Caporaso, N.E., Katki, H.A., et al. (2010) C-Reactive Protein and Risk of Lung Cancer. Journal of Clinical Oncology, 28, 2719-2726. https://doi.org/10.1200/JCO.2009.27.0454

[25] Heikkilä, K., Silander, K., Salomaa, V., et al. (2011) C-Reactive Protein-Associated Genetic Variants and Cancer Risk: Findings from FINRISK 1992, FINRISK 1997 and Health 2000 Studies. European Journal of Cancer, 47, 404-412. https://doi.org/10.1016/j.ejca.2010.07.032

[26] Siemes, C., Visser, L.E., Coebergh, J.W., et al. (2006) C-Reactive Protein Levels, Variation in the C-Reactive Protein Gene, and Cancer Risk: The Rotterdam Study. Journal of Clinical Oncology, 24, 5216-5222. https://doi.org/10.1200/JCO.2006.07.1381

[27] Allin, K.H., Nordestgaard, B.G., Zacho, J., et al. (2010) C-Reactive Protein and the Risk of Cancer: A Mendelian Randomization Study. Journal of the National Cancer Institute, 102, 202-206. https://doi.org/10.1093/jnci/djp459

[28] Lin, N., Li, J., Ke, Q., et al. (2020) Clinical Significance of C-Reactive Protein to Albumin Ratio in Patients with Hepatocellular Carcinoma: A Meta-Analysis. Disease Markers, 2020, Article ID: 4867974. https://doi.org/10.1155/2020/4867974 
[29] Liu, Z., Jin, K., Guo, M., et al. (2017) Prognostic Value of the CRP/Alb Ratio, a Novel Inflammation-Based Score in Pancreatic Cancer. Annals of Surgical Oncology, 24, 561-568. https://doi.org/10.1245/s10434-016-5579-3

[30] Ni, X.F., Wu, J., Ji, M., et al. (2018) Effect of C-Reactive Protein/Albumin Ratio on Prognosis in Advanced Non-Small-Cell Lung Cancer. Asia-Pacific Journal of Clinical Oncology, 14, 402-409. https://doi.org/10.1111/ajco.13055

[31] Wu, J., Tan, W., Chen, L., et al. (2018) Clinicopathologic and Prognostic Significance of C-Reactive Protein/Albumin Ratio in Patients with Solid Tumors: An Updated Systemic Review and Meta-Analysis. Oncotarget, 9, 13934-13947. https://doi.org/10.18632/oncotarget.24172

[32] Xu, S., Li, X., Liu, Y., et al. (2019) Inflammasome Inhibitors: Promising Therapeutic Approaches against Cancer. Journal of Hematology \& Oncology, 12, 64. https://doi.org/10.1186/s13045-019-0755-0 